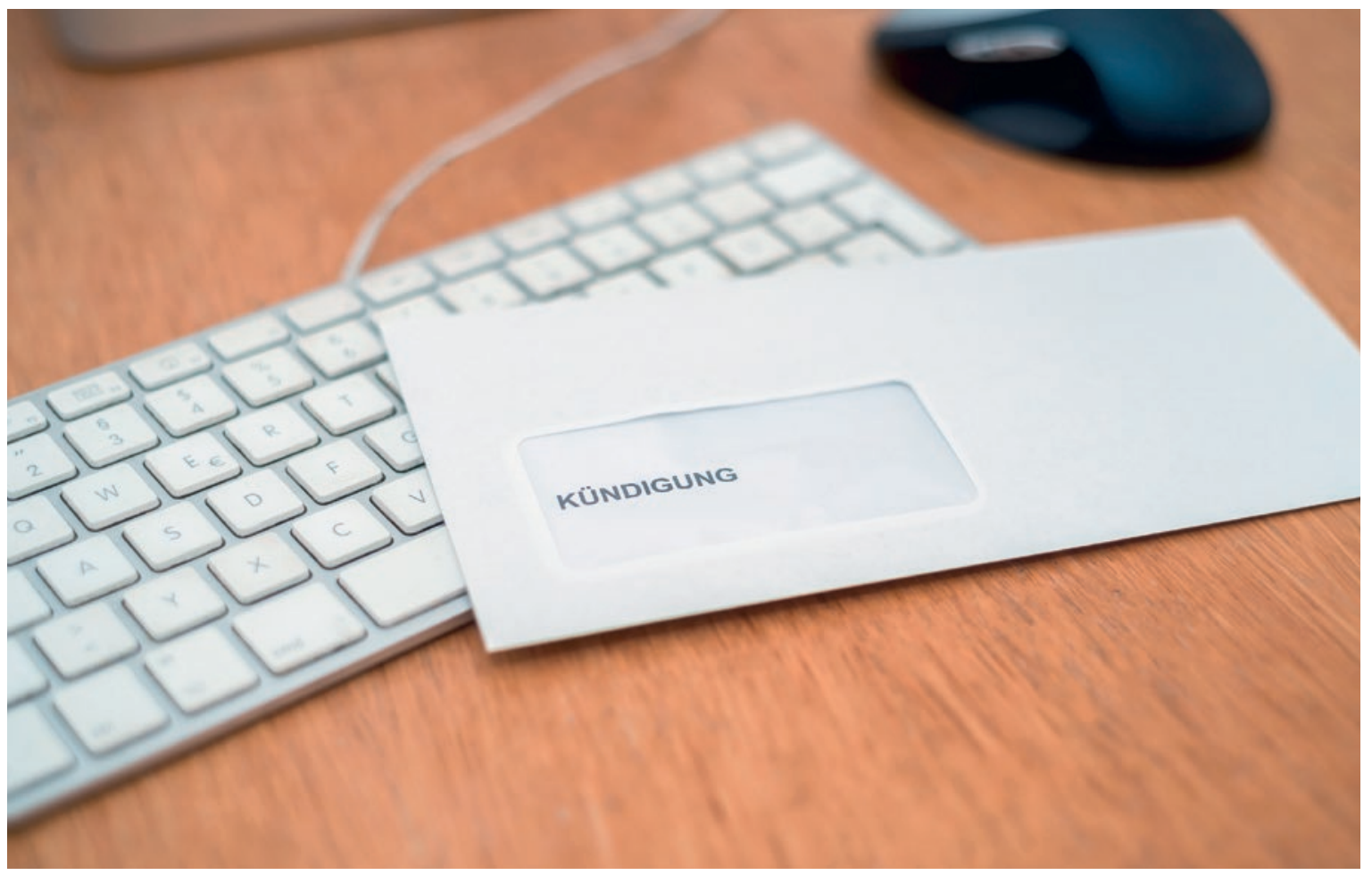

Die aktuellen Entlassungen von Chefärztinnen und -ärzten sind Ausdruck ungelöster Probleme in der Spitalkultur.

\title{
Chefärzte entlassen
}

\section{ist keine Lösung}

\section{Beat Frauchiger ${ }^{a, c}$ und Markus Schmidli ${ }^{b, c}$}

a Prof. Dr. med., Facharzt für Innere Medizin und Angiologie, Senior Consultant; ${ }^{b}$ Dr. med., Facharzt für Innere Medizin und Nephrologie, Leiter allgemeine

Innere Medizin a.i., Spital Appenzell; ${ }^{c}$ Chefarzt i.R.

Die aktuellen Konflikte mit Chefärzten und Chefärztinnen wurzeln in Verwerfungen des Gesundheitswesens. Der Chefarzt ist dem gesellschaftlichen und medizinischen Wandel, überbordender Bürokratie und ungelösten Fragen der Entwicklung unserer Spitäler besonders ausgesetzt. Ohne Besinnung auf Wertschätzung wird sich der systemrelevante Mangel an guten Chefärztinnen akzentuieren.

Nach jahrelangem journalistischem Sperrfeuer gegen Spitalärzte ist Erstaunliches passiert. Zwei Trennungen von Chefärzten im Kanton Zürich führten zu einem kleinen Tsunami in der Tagespresse mit grossen Sympathien für die Entlassenen. Das wiederum veranlasste die Spitaldirektoren der Schweiz, um mediale Zurückhaltung zu bitten, zu komplex sei die Materie als Objekt öffentlicher Diskussion [1]. Wirklich? Der CEO des Kantonsspitals Baden entgegnete, dass die dauernde mediale Verunglimpfung zu Nachwuchsproblemen bei Chefärzten führen könnte [2]. Das bringt es auf den Punkt. Es wird immer schwieriger, gute Chefärztinnen zu finden, und diese Entwicklung gibt Anlass zu grosser Sorge.

\section{Schlagzeilen und Realität}

Das so gerne bediente Klischee der geld- und machtbewussten Halbgötter in Weiss ist realitätsfern. Die Autoren haben jahrzehntelang als Chefärzte gearbeitet und kennen das Schweizer Gesundheitswesen auch aus ihren anderen Funktionen. Ihre Wahrnehmung ist, dass 
die Konflikte mit Chefärzten nicht in narzisstischer Fehlinterpretation des Berufsbildes - Ausnahmen ausgenommen - wurzeln. Vielmehr sind sie Manifestation vieler ungelöster Probleme im Gesundheitswesen. Unantastbare Chefärztinnen und Chefärzte - das war vielleicht einmal. Heute fragt sich der junge Mediziner: Will ich mir das wirklich antun? Diese Entwicklung ist fatal, weil gute Chefärztinnen den medizinischen Alltag entscheidend prägen.

\section{Kein Auslaufmodell}

Das Wort «systemrelevant» trifft auf Chefärztinnen und -ärzte mehrfach zu. Sie arbeiten direkt am Krankenbett, können durch geschickte Medizin in der Klinik die knappen Finanzen optimal nutzen und ihre Mitarbeitenden prägen. Hochgerechnet auf 15-20 Jahre Berufstätigkeit ist der fachliche und ökonomische Einfluss hoch. Sie sind Leader einer Klinik - diese hat sich im Spital während Jahrzehnten als effiziente Organisationseinheit bewährt. Kritische Berichte monieren oft hierarchische Strukturen.

\section{Chefärztinnen formen den ärztlichen Nach-} wuchs - eine Aufgabe mit grossen Auswirkungen auf die künftige medizinische Versorgung.

Für 30-50\% der Mitarbeitenden einer Klinik ist aber die Tätigkeit Teil ihrer Ausbildung. In dieser Umgebung gehören Kontrolle und Korrektur dazu. Standard ist heute Teamarbeit mit klaren Verantwortungen, ohne ätzenden Kommandoton. Der Einsatz der Chefärztin ist nicht nur hoch, sondern sehr direkt «an der Front». Sie ist mitten in der Nacht auf der Notfallstation oder im Operationssaal und nimmt als behandelnde Ärztin persönlich Einfluss auf den Krankheitsverlauf. Der 24-Stunden/7-Tage-Betrieb für den Patienten ist sehr spezifisch für diesen Beruf - auch im

\section{Die Autoren haben Kenntnis von mindestens} 30 vorzeitigen Abgängen von Chefärzten in den letzten fünf Jahren.

Spital. Ökonomen leisten keine Dienste, in der Pflege ist ab der zweiten Hierarchiestufe Dispens vom Notfall- und Nachtdienst traditionelles Privileg. Schliesslich formen Chefärztinnen auch den ärztlichen Nachwuchs - eine Aufgabe mit grossen Auswirkungen auf die zukünftige medizinische Versorgung.

Da die Klinik kein Auslaufmodell ist, werden wir auch weiterhin Chefärzte brauchen. Übrigens: Trotz schwierigem Umfeld arbeiten auch heute Chefärztinnen als begeisterte und begeisternde Chefinnen, wenn Chemie und Wertschätzung stimmen. Sie sind die Anker für die Motivation fähiger Nachwuchskräfte.

\section{Zunehmende Belastungen}

Die Autoren haben Kenntnis von mindestens 30 vorzeitigen Abgängen von Chefärzten in den letzten fünf Jahren. Ohne fundierte Kenntnisse der Trennungsgründe stellen wir fest, dass es zunehmend Abgänge von fähigen Chefärzten gibt, die frustriert sind, die «Nase voll haben», denn Anspruch und Wirklichkeit im

\section{Weder in der Verwaltung noch in der Wirt-} schaft finden sich Führungspositionen, deren Breite die der chefärztlichen Funktion erreicht.

Alltag driften zunehmend auseinander. Die Stellenausschreibungen sind Mantra-ähnlich. Gesucht wird eine Persönlichkeit sowohl mit Fachwissen, Führungs- und Organisationskompetenz, Flair für Teamwork als auch Verständnis und Adhärenz an ökonomische Auflagen, hoher Menschlichkeit und Visionen für die Klinik und das Spital. Die flapsige Umgangssprache bezeichnet dies als «eierlegende Wollmilchsau». Weder im Verwaltungs- noch im Wirtschaftsbereich finden sich Führungspersonen, deren Tätigkeit die Breite der chefärztlichen Funktion erreicht. Nebst den Grundaufgaben ist der enorme gesellschaftliche und medizinischtechnische Wandel eine zunehmende Belastung. Das «Management der allseitigen Anspruchshaltungen» ist sehr aufwendig. Eine weitere grosse Baustelle ist das ungebremste Wachstum administrativer Aufgaben. Je nach Klinikgrösse beträgt die patientenferne Arbeit 40-60\%, ohne dass ein echter Nutzen nachgewiesen werden kann.

Diese Entwicklungen machen die Tätigkeit als Chefärztin oder -arzt zunehmend unattraktiv; parallel dazu hat die Arbeit in Privatpraxen und -spitälern an Anziehungskraft gewonnen. Ein anderer Arbeitsrhythmus und lukrative Tarife, speziell für technische Diagnose oder Therapie, ermöglichen ausserhalb öffentlicher Spitäler ein weniger anstrengendes Leben. Die Frustration ist real: Eine Umfrage des Vereins Leitender Spitalärzte bestätigt, dass rund 25\% der aktuellen Chefärztinnen und Chefärzte Veränderungsabsichten hegen [3]. Wer in Chefarztwahlen involviert war, kennt das Phänomen: Meldeten sich vor 25 Jahren noch 20 Bewerberinnen und Bewerber für eine chefärztliche Stelle, sind es heute noch fünf, und davon kommen vielleicht drei wirklich in Frage für eine nähere Prüfung des Dossiers. 


\section{Im Konfliktfeld}

In dieser Spitallandschaft lassen sich Konflikte nicht vermeiden. Ein Chefarzt muss für seine Positionen und seine Klinik kämpfen. Sich von einer Chefärztin zu trennen, wenn die fachlichen Leistungen stimmen, die Patientinnen, Mitarbeitenden und Zuweisenden zufrieden sind, birgt ein hohes Risiko für einen Reputationsschaden, bedeutet aber auch einen materiellen Verlust. Meist hat die Entlassung noch eine Reihe weiterer Abgänge zur Folge - der Wiederaufbau einer stabilen Klinik dauert Jahre. Hire and Fire mag in der Industrie angehen. In der Klinikführung ist Kontinuität für die Qualität sehr zentral. Die Bereitschaft, Konflikte auszuhalten, ist leider (allseits) markant gesunken und fördert respektlose Beschlüsse, statt konsequent alle Möglichkeiten der Unterstützung und Wertschätzung auszuschöpfen.

\section{Die Systemkrise}

Medizin und Ökonomie sollen zusammen mit Erfolg möglich sein - diesem Schlusssatz der Spitaldirektoren ist zuzustimmen. Bei der Problemlösung gehen die Meinungen allerdings oft auseinander. Die Spitaldirektoren vertreten in der Regel die Vorgaben der Spitalträger. Unsere Meinung ist: Sie könnten sich durchaus häufiger auf die Seite der patientennahen Ärzte und Pflege schlagen. Dies gilt für den Kampf gegen die überbordende Bürokratie, aber auch gegen den enormen Reformstau, der der Politik anzulasten ist. Das Anlegen neuer Datenfriedhöfe löst die wirklich drängenden Fragen nicht. Es geht letztlich darum, dass immer mehr möglich wird, aber speziell bei der medizini-

Prof. Dr. med.

Beat Frauchiger

Chefarzt i.R./

Senior Consultant

Facharzt für Innere Medizin

und Angiologie

Amselweg 3

CH-8500 Frauenfeld

beat.frauchiger[at]stgag.ch

Dr. med. Markus Schmidli

Chefarzt i.R./Leitung

allg. Innere Medizin a.i.

Spital Appenzell

Facharzt für Innere Medizin

und Nephrologie

Alpenrose 949

CH-9103 Schwellbrunn

markus.schmidli[at]svar.ch

\section{Das Wichtigste in Kürze}

- Die Autoren sehen beim Gesundheitssystem in seinem aktuellen Zustand einen drohenden Mangel an fachlich guten Chefärztinnen und -ärzten.

- Die Anforderungen an Personen in einer chefärztlichen Position sind immens: Fachwissen, Führungskompetenz, Managementskills, Flair fürTeamwork, hohe Menschlichkeit und ein innovativer Geist werden vorausgesetzt.

- Die Autoren regen an, dass sich die Spitaldirektionen wieder vermehrt mit der Situation der patientennahen Berufsgruppen auseinandersetzen sollten, anstatt mehrheitlich aufseiten der Spitalträgerinnen und -träger zu agieren. schen Behandlung älterer Menschen längst nicht mehr alles sinnvoll ist und sich die Grenzen des Finanzierbaren zeigen. Die Formulierung im Inserat, dass «erst ein finanziell gesundes Spital eine qualitativ gute Medizin und vice versa ermöglicht», klingt blumig. Aber was nützt die Zuführung von mehr Wasser, wenn das Loch im Fass immer grösser wird? Wenn die Leute immer

Bevor Entlassungen ausgesprochen werden, sollten alle Möglichkeiten der Unterstützung und Wertschätzung ausgeschöpft werden.

älter werden, immer mehr Medizin benötigt, diese immer aufwendiger und teurer wird und wir immer weniger Personal finden, das in diesem System mitmachen will? Dazu fehlen vernünftige Strategien. Mitten in diesen unbeantworteten Fragen arbeitet die Chefärztin und muss versuchen, dem Patienten, der fachlichen Vorgabe, den Mitarbeitenden und den Finanzen gerecht zu werden. Dieser Spagat überfordert zunehmend, nicht nur den Chefarzt. Die Pflege sendet ähnliche Signale aus. Man kann Chefärztinnen und -ärzte entlassen, das Problem aber wird grösser. Wie heisst es doch: den Sack geschlagen, statt den Esel.

\section{Literatur}

1 Medizin und Oekonomie: Gegeneinander oder Miteinander? Neue Zürcher Zeitung S. 14, 10. Oktober 2020

2 Chefärzte verdienen Respekt. Neue Zürcher Zeitung, S. 18, 6 November 2020.

3 Umfrage unter den Mitgliedern VLSS. Info Chefärzte und Leitende Spitalärzte Schweiz S. 6, 22. April 2020.

Bildnachweis

(c) Thorsten Schiller | Dreamstime.com, Symbolbild

\section{L'essentiel en bref}

- Selon les auteurs, le système de santé va, dans son état actuel, vers une pénurie imminente de bons spécialistes au poste de médecins-chefs.

- Les exigences imposées aux personnes occupant un poste de médecin-chef sont énormes: connaissances spécialisées, compétences en matière de leadership, aptitudes à la gestion, sens du travail en équipe, compétence humaine et esprit d'innovation.

- Les auteurs suggèrent que les directions des hôpitaux devraient davantage s'occuper de la situation des professions proches des patients au lieu d'intervenir en premier lieu auprès des instances en charge des hôpitaux. 